DE93 002097

\title{
Reactive Fluid Flow Models and Applications to Diagenesis, Mineral Deposits and Crustal Rocks: A Progress Report
}

\author{
Antonio C. Lasaga and Danny M. Rye
}

This project is obtaining new results and developing new techniques along three directions: a) experimental studies of water-rock reactions b) theoretical modeling of coupled fluid flow-chemical reactions and c) isotopic measurements of both regional isotopic compositions as well as isotopic zoning within individual mineral grains. An important part of the project is the integration of all three approaches into a concerted effort aimed at new understanding of the behavior of fluids and their chemical reactions with minerals in the crust.

The experimental work pioneered in our laboratories has produced several startling results on the kinetic rate laws of silicate-water reactions. The approach to equilibrium has been shown to follow a non-linear path in rate constant-free energy space. This behavior is quite distinct from most work done ty geochemists on modeling silicate behavior in diagenesis, weathering, hydrothermal systems or environmental models. The work to date has involved albite, kaolinite and. gibbsite, which together with silica would comprise a kinetic "granite system prototype".

The theoretical modeling has produced a state-of-the-art computer code that can efficiently handle dozens of chemical species, many mineral reactions and variations of fluid flow properties and temperature in both one and two dimensions. In addition the code can now treat oxidation-reduction reactions and isotopic exchange between fluids and minerals.

The main thrust of the theoretical modeling has been to develop further the differences between equilibrium, steady state, and non-steady-state behavior of the chemical evolution of open fluid-rock systems. These differences have not been fully appreciated in previous models. For example, mineral-water systems which would attain equilibrium rather quickly without fluid flow, can achieve a steady state which exhibits significant deviationi from equilibrium in the case that there is fluid flow. Furthermore, fundamental differences arise between one-dimensional and two-dimensional treatments of cimilar systems. In particular, we have found that the mixing of fluids in two dimensional models (from processes such as dispersion) has profoundly diffe:ent chemical patterns from those found in the one dimensional models.

To apply the models to field data we have developed techniques to measure isotopic zonation within individual mineral grains. These techniques include: 1) micro-drilling of samples with a fine dental drill coupled with a micro volume inlet system on the mass spectrometer. 2) separation of minerals from fine grain rocks hy a series of dissolution steps. These techniques are superior to the laser technique's 
because they avoid the problem of isotopic fractionation during the sampling process, and they allow us to analyze more than one mineral phase in the rock.

We are presently applying the two-dimensional code 2DREACT to the study of reactive flow in hydrothermal systems. The code represents a new approach to the modeling of simultaneous reaction and transport which was completed in the first months of this project. The code calculates heat transfer and hydrodynamic flow in a convecting hydrothermal system which are then coupled to the calculations of reactive transport. The coupling of heat transfer, fluid flow, and mass transport with the chemical reactions, therefore, give a physically realistic description of the rate at which rock alteration occurs in a transient hydrothermal system. The code has been run with as many as 42 aqueous species (13 of which are actually independent), 18 minerals and 800 grid points, although most runs have been carried out with 400 grid points. We have carried out the simulations for as long as 50,000 years using time stops ranging up to 5 years, a feat which may not be possible without the use of the implicit code we have developed.

The code 2DREACT employs a kinetic formulation for all of the mineral-water reactions and assumes equilibrium for the reactions between aqueous species. Another novel feature which was completed in the early months of the project is full basis switching within the finite difference scheme. A new method was developed whereby it is possible to write the reactions at each grid point in terms of the dominant species (and the dominant redox couple) and to correct the fluxes between adjacent grid points where the reactions are written using different basis sets. This innovation gives substantially improved numerical stability and rates of convergence compared to schemes which do not use basis switching.

The simulations which are in progress now track the transient evolution of the thermal field, hydrodynamic flow regime, and mineral-water reactions during the startup of a hydrothermal system. These simulations include 42 aqueous species and 18 minerals in order to give an approximately realistic description of what happens when a convection cell is initiated in granitic rock. Our results indicate that substantial reaction occurs before a hydrodynamic steady state is attained. This observation is particularly important because the reactions may result in a change in the permeability of the medium. We are studying this problem by comparing simulations with and without porosity/permeability change due to mineral dissolution and precipitation reactions. The results are very sensitive to the initial diameter of the fractures in the rock (i.e., the hydraulic radius), but as an example, for an initial porosity of $1 \%$ and a permeability of $3 \mathrm{mD}$, we find an order of magnitude increase in the permeability of the medium in the initial stages of the hydrothermal system even over relatively short time scales.

We have also included stable isotope reactions in the code 2DREACT. The primary purpose of this addition was to use the code to assess the validity of various schemes for computing water-rock ratios based on either isotopic or mineralogic reaction progress in paleo-hydrothermal systems. The calculations are useful in 
evaluating the water rock indicators because they represent a self-consistent simulation of infiltration-driven reactions where both the actual fluid fluxes and the resulting changes in modal mineralogy in the rock (albeit a synthetic one) are available. This exercise has been carried out in a series of simulations for the Butte hydrothermal system and it gives us, for the first time, an estimate of the errors accompanying calculations of water-rock ratios using the standard methods.

As a first step in using isotopic data in metamorphic systems, we have developed one-dimensional isothermal steady state flow path isotopic exchange models for both veins and wall rocks. These models incorporate diffusion, advection, and isotopic exchange (assuming first order kinetics). We find that even for relatively rapid isotopic exchange rates and small fluid fluxes significant oxygen isotope disequilibrium between fluid in a crack and wall rock can be maintained for long distances for long periods of time along the flow path.

By combining the results of our exchange models with paragenetic studies and isotopic measurements we have established a fluid history for the Wepawaug Schist of South-central Connecticut. The salient points are that there was an early low temperature pervasive exchange that required an interconnected pore fluid up to about $300{ }^{\circ} \mathrm{C}$. Above $300^{\circ} \mathrm{C}$ there was not an interconnected pore fluid and fluid transport occurred in cracks during transient events.

Constraints on the amount of fluid present at low grades comes from the relationship of the isotopic systematics between metacarbonates and the surrounding metapelites. Small amounts of metacarbonate are found at all metamorphic grades. Regardless of metamorphic grade, the metacarbonates have a nearly uniform whole rock isotopic composition. The whole rock oxygen isotopic values are several permil to small to represent original isotopic compositions. The implication is that all the carbonates underwent massive isotopic exchange with the surrounding pelites at about the same conditions. The whole rock oxygen isotopic values preserved in the metacarbonates require that this exchange took place at or below $300{ }^{\circ} \mathrm{C}$. This exchange could not have taken place without an interconnected pore fluid. The preservation of high temperature isotopic fractionations between product minerals in the metacarbonates requires that this exchange occurred during the prograde path of the metamorphism.

The transport exchange model can also be used to put constraints on how much or how long fluid was present under high grade metamorphic conditions. The preservation of fine-scale isotopic gradients at metacarbonate-vein margins, and the preservation of isotopic disequilibrium between reactant and product phases within metacarbonate units in the Wepawaug Schist require that interconnected pore fluids occupied less than 0.03 percent of the available grain boundary regions or were present for less than 10 to 1000 years at temperatures above $300^{\circ} \mathrm{C}$..

We have used the transport-exchange model to interpret a set of oxygen isotope data from veins, that were produced near the peak of metamorphism $\left(>500^{\circ} \mathrm{C}\right)$, and 
surrounding wall rocks. The transport-exchange model successfully reproduces the field data for fracture-controlled flow with a time integrated flux of $10^{2}$ to $10^{4} \mathrm{~m}^{3}$ fluid $/ \mathrm{m}^{2}$ and a distance along the steady state gradient, at which one-half of isotopic equilibrium with wall rocks was achieved, of $1100 \mathrm{~m}$.

We are presently developing two and three ciimension isotopic transport-flow models that can be applied to field data from several localities. We are gathering field data on the grain-grain scale, the outcrop scale, and on the metamorphic complex scale. As a first step we are measuring oxygen and carbon isotope ratios on micro samples of single calcite crystals from marbles that are in contact with pelitic rocks. The micro samples are taken with a high speed micro dental drill. The $\mathrm{CO}_{2}$ generated by reaction with phosphoric acid is measured on the mass spectrometer using our micro gas handling system. With these dental drill samples we are, for the first time, determining the pattern of isotopic zoning across single crystals of calcite. This study is giving us a unique set of data that, as we develop the theory, will allow us to put severe constraints on the fluid flux during the growth of calcite crystals within the marbles studied.

In order to be able to interpret these new data, the fully coupled chemical reaction and flow model has been modified to take into account changes in the isotopic composition of the fluid and of individual minerals as a function of time and space. We have already tested the code with $\delta^{18} \mathrm{O}$ calculations (see enclosed paper). The work here has begun to pioneer the quantitative treatment of chemical transport and reactions along a vein. In particular, the results focus on the major conceptual changes that take place in the transition from a one dimensional model of vein alteration (e.g. wall rock alteration perpendicular to the vein) to a two dimensional model, which can follow the evolution of the fluid along the vein as well as the movement and alteration perpendicular to the vein.

A new area of investigation of direct relation to this project is the analysis of the major bauxite deposit in the Pijiguaos district of Venezuela $(500 \mathrm{Km}$ scuth of Caracas and $35 \mathrm{Km}$ from the Orinoco river). The Venezuelan company, C.V.G. Bauxita Venezolona, C.A. (BAUXIVEN) has agreed to turn over the results of more than 100,000 analysis of extensive drilling in the deposit covering a large surface area. Each drill core ranges from soil surface to the parent rock, the Precambrian Parguaza granite. Furthermore, current studies by the Universidad Central de Venezuela will focus on the variation in solution composition in the present bauxite deposit. We have been collaborating with the group in the Universidad Central and will be involved in the design of the field experiments being planned. This deposit, aside from its significant economic importance to Venezuela, provides a unique opportunity to test not only our fluid flow code but also the isotope zonation experimental techniques and models as well as the detailed thermodynamic and kinetic studies that we have been and continue to carry out in the system, feldspar-kaolinite-gibbsite-silica. 


\section{TITLES OF COMPLETED WORK SUPPORTED BY GRANT AND ACKNOWLEDGED}

Lasaga, Antonio C., and Rye, Danny M.,1992, Fluid Flow and Chemical reaction Kinetics in Metamorphic systems, in press, American Journal of Science

Steefel, Carl I., and Lasaga, Antonio C., 1992, Putting Transport into Water-Rock Interaction Models, submitted, Geology

Nagy, K.L., and Lasaga, A.C., 1992, Dissolution and Precipitation Kinetics of Gibbsite at $80^{\circ} \mathrm{C}$ and $\mathrm{pH}$ 3: The Dependence on Solution Saturation State, in press,

Steefel, C.I.,1991, Coupled reaction-transport models: Development and application to geological systems. PhD Dissertation, Yale University.

Palin, J. Michael, 1992, Petrologic and Stable Isotopic Studies of the Wepawaug Schist, Connecticut. PhD Dissertation, Yale University. 

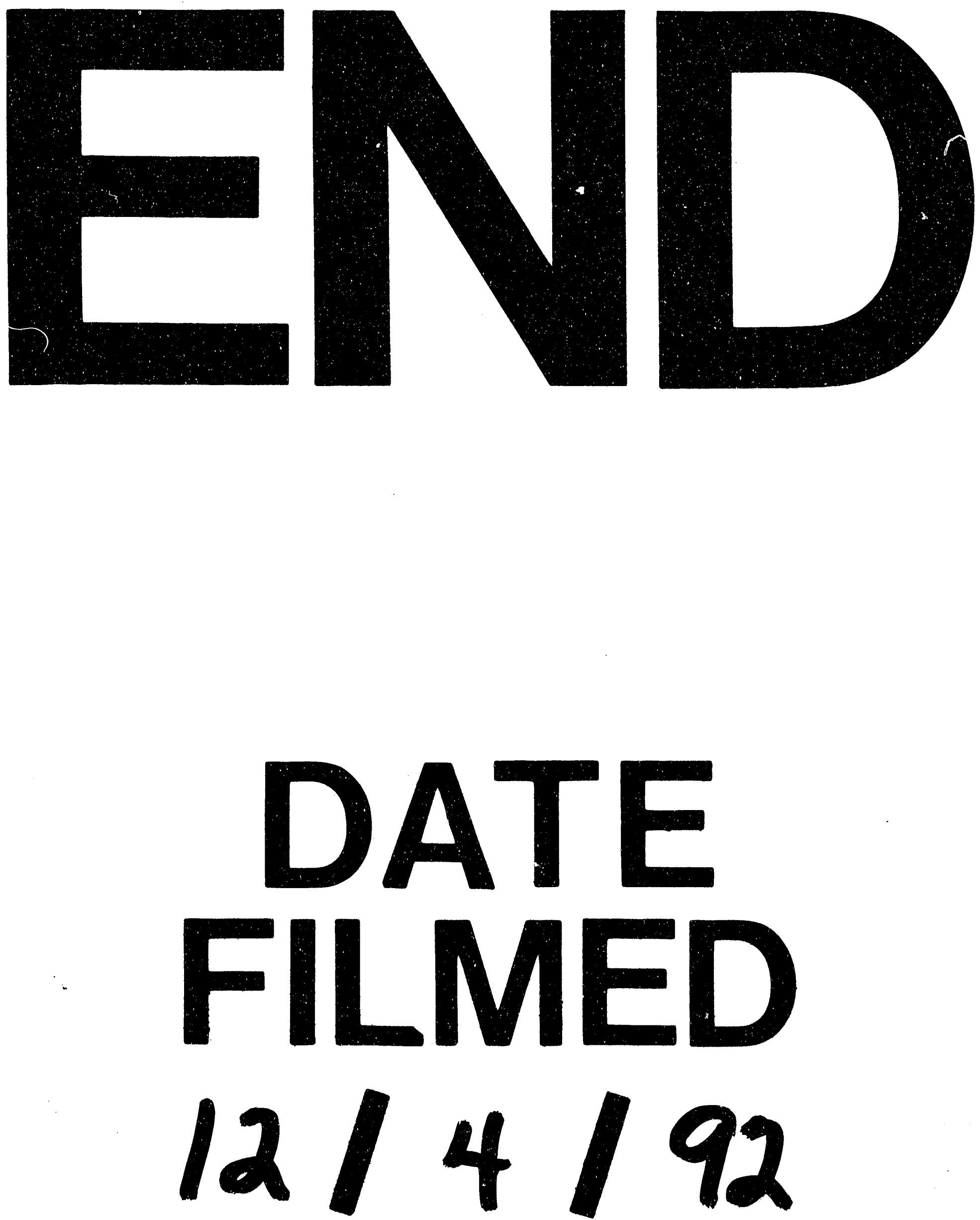
